\title{
Strength and Nature of Host-Guest Interactions in Metal-Organic Frameworks from a Quantum-Chemical Perspective
}

\author{
Michelle Ernst, ${ }^{* a, b}$ Ganna Gryn'ova*a,b \\ ${ }^{a}$ Computational Carbon Chemistry Group, Heidelberg Institute for Theoretical Studies (HITS gGmbH), \\ 69118 Heidelberg, Germany. \\ ${ }^{b}$ Interdisciplinary Center for Scientific Computing, Heidelberg University, 69120 Heidelberg, Germany \\ *E-mail:michelle.ernst@h-its.org,ganna.grynova@h-its.org
}

\begin{abstract}
Metal-organic frameworks offer a convenient means for capturing, transporting, and releasing small molecules. Rational design of such systems requires an in-depth understanding of the underlying non-covalent interactions, and the ability to easily and rapidly pre-screen candidate architectures in silico. In this work, we devised a recipe for computing the strength and analysing the nature of the host-guest interactions in MOFs. Using experimentally characterised complexes of calcium-adipate framework with 4,4'-bipyridine and 1,2-bis(4-pyridyl)ethane guests as test systems, we have assessed a range of density functional theory methods, energy decomposition schemes, and non-covalent interactions indicators across realistic periodic and finite supramolecular cluster scales. We find that appropriately constructed clusters readily reproduce the key interactions occurring in periodic models at a fraction of the computational cost and with an added benefit of diverse density partitioning schemes. Host-guest interaction energies can be reliably computed with dispersioncorrected density functional theory methods; however, decoding their precise nature demands insights from energy decomposition schemes and quantum-chemical tools beyond local bonding indices (e.g., the quantum theory of atoms in molecules), such as the non-covalent interactions index and the density overlap regions indicator.
\end{abstract}




\section{Introduction}

Increasingly complex molecular structures have been developed to satisfy the ever-growing demand for highly efficient, multi-functional, and environmentally conscious materials. Among these, metal-organic frameworks (MOFs) ${ }^{1,2}$ are attracting growing attention due to their highly tuneable composition, topologies, and physico-chemical properties. MOFs are porous crystalline hybrid inorganic-organic compounds that consist of regularly connected nodes and linkers, have high internal surface areas and low densities, and, most importantly for practical purposes, are able to absorb small guest molecules. The associated physi- and chemisorption via host-guest interactions depend on the nature of the guest molecule, pore size and volume, surface area, solvent, etc. Numerous applications of MOFs hosting small molecules include gas storage and separation, ${ }^{3,4}$ drug delivery, ${ }^{5}$ (photo-)catalysis, ${ }^{6,7}$ and biological imaging. ${ }^{8-10}$

Synthesis and characterisation of existing systems, as well as design and pre-screening of new and improved candidates require coordinated efforts from experiment and simulation. The myriad permutations of nodes and linkers into MOFs with distinct topologies, able to encapsulate molecular guests at varying loadings, require theory to rationally guide practice. Among the most commonly employed techniques to model the host-guest interactions in MOFs are grand-canonical Monte-Carlo (GCMC) and molecular dynamics (MD) simulations. The former are particularly well-suited for weakly interacting systems, such as gases in storage and catalysis applications, ${ }^{11-14}$ but are also widely used to model the loading capacity of drug-delivering MOFs; ${ }^{15-17}$ the latter are often employed to simulate diffusion of guests inside the pores. ${ }^{16,18}$ These studies demonstrate not only the utility of GCMC and MD simulations to capture the structural features of MOFs, but also the necessity to include quantum-chemical effects at the ab initio or density functional theory (DFT) level in order to explicitly examine the host-guest interactions.

However, modelling the intake of small molecules within MOFs using DFT and post-HartreeFock (post-HF) methods faces several challenges. First, MOFs are periodic systems that typically feature 'computationally expensive' large unit cells with several metal atoms. Second, reliable experimental structures of the host-guest complexes that can serve as a starting point in subsequent geometry relaxation are rarely available. In such cases, a guess for the starting geometry can be generated using, for example, electrostatic complementarity. ${ }^{19}$ Third, MOFs alone and, in particular, their complexes with small molecules are often disordered and rather dynamic. Consequently, most examples applying DFT and post-HF methods to MOFs focus on the uptake of very small compounds (e.g., gases and volatiles). ${ }^{20-29}$ Periodic DFT studies on MOFs with larger guest molecules are somewhat less common. ${ }^{19,30,31}$ 
As an alternative to periodic approaches, the so-called 'cluster' computations focus on a finitesize model of the host-guest complex containing the interaction regions between the molecule and the MOF. Such a drastic decrease in the system size and conformational space opens the doors to more accurate computations of complex geometries and interaction energies. ${ }^{32-34}$ Moreover, it provides access to a broad range of sophisticated in silico techniques for quantifying, conceptualising, and visualising non-covalent interactions, ${ }^{35}$ which, nonetheless, remain underutilised in MOF studies. ${ }^{36}$ These quantum-chemical tools employ schemes for partitioning the multidimensional wavefunction and the total electron density into localised regions, as well as for decomposing the total interaction energy into physically meaningful components. Nowadays, these approaches are commonplace in studies on chemical stability and reactivity, ${ }^{37-41}$ catalysis, ${ }^{42-44}$ biomolecular interactions, ${ }^{45,46}$ molecular crystals, ${ }^{47-49}$ and organic electronics. ${ }^{50-52}$

Due to the aforementioned scarcity of accurate host-guest complex geometries, a profound theoretical understanding of the microscopic building blocks and their interactions is crucial for the rational design of new MOFs with desired absorption properties. In this study, we probe the applicability and validity of diverse quantum-chemical tools for analysing and visualising the strength and physical nature of the non-covalent interactions in the MOF host-guest complexes across periodic and finite-size scales.

\section{Methodology}

\section{Studied systems}

A MOF composed of nodes with calcium and oxygen atoms and adipate linkers was selected due to the availability of accurately determined structures of its host-guest complexes. ${ }^{53}$ Two host-guest complexes were considered: system 1 (CCDC name: PARHAS), containing 4,4'-bipyridine, and system 2 (CCDC name: PARHEW), containing 1,2-bis(4-pyridyl)ethane. Both systems crystallise in the monoclinic P21/c space group, but 2 has a slightly larger volume of $1934.94 \AA^{3}$ compared to $1752.46 \AA^{3}$ for $\mathbf{1}$. This is a consequence of the sizes of the guest molecules used as templates during synthesis. The framework is connected by adipate linkers in the $a$ and $b$ directions and by calcium and oxygen atoms in the $c$ direction. The templating guest molecules reside in channels along the $c$ direction. Each guest forms two distinct (not symmetry-related) hydrogen bonds with the host framework, in which the pyridinic nitrogen of the guest molecule is the hydrogen acceptor and the water molecule coordinated to the calcium of the framework is the hydrogen donor (Figure 1). 


\section{Computational methods}

Constrained optimisation of the water molecules involved in the hydrogen bonds was performed to address the inaccurate assignment of the hydrogen atom positions in standard X-ray diffraction experiments (see ESI for further discussion). Geometry optimisations were performed at the B3LYPD3 $3^{54} /$ pob-TZVP_rev $2^{55}$ level of theory in a periodic setting with the shrinking factor set to 6 using Crystal17. ${ }^{56}$ Interaction energies for the studied host-guest complexes in so-obtained geometries were computed using periodic DFT with several functionals, namely B3LYP-D3, PBE0-D3, PBE-D3, LC$\omega$ PBE-D3, M06-D3, and M06-2X-D3:

$\Delta E=E_{h g}-\left[E_{h}+E_{g}\right]$

where $E_{h g}$ is the energy of the relaxed host-guest complex, and $E_{h}$ and $E_{g}$ are energies of the isolated host and guest molecules in their crystal geometries, respectively. Finally, the topology of the electron density in periodic computations was analysed in the framework of Bader's Quantum Theory of Atoms in Molecules (QTAIM) ${ }^{57}$ using TOPOND ${ }^{58}$ as implemented in Crystall7. ${ }^{56}$

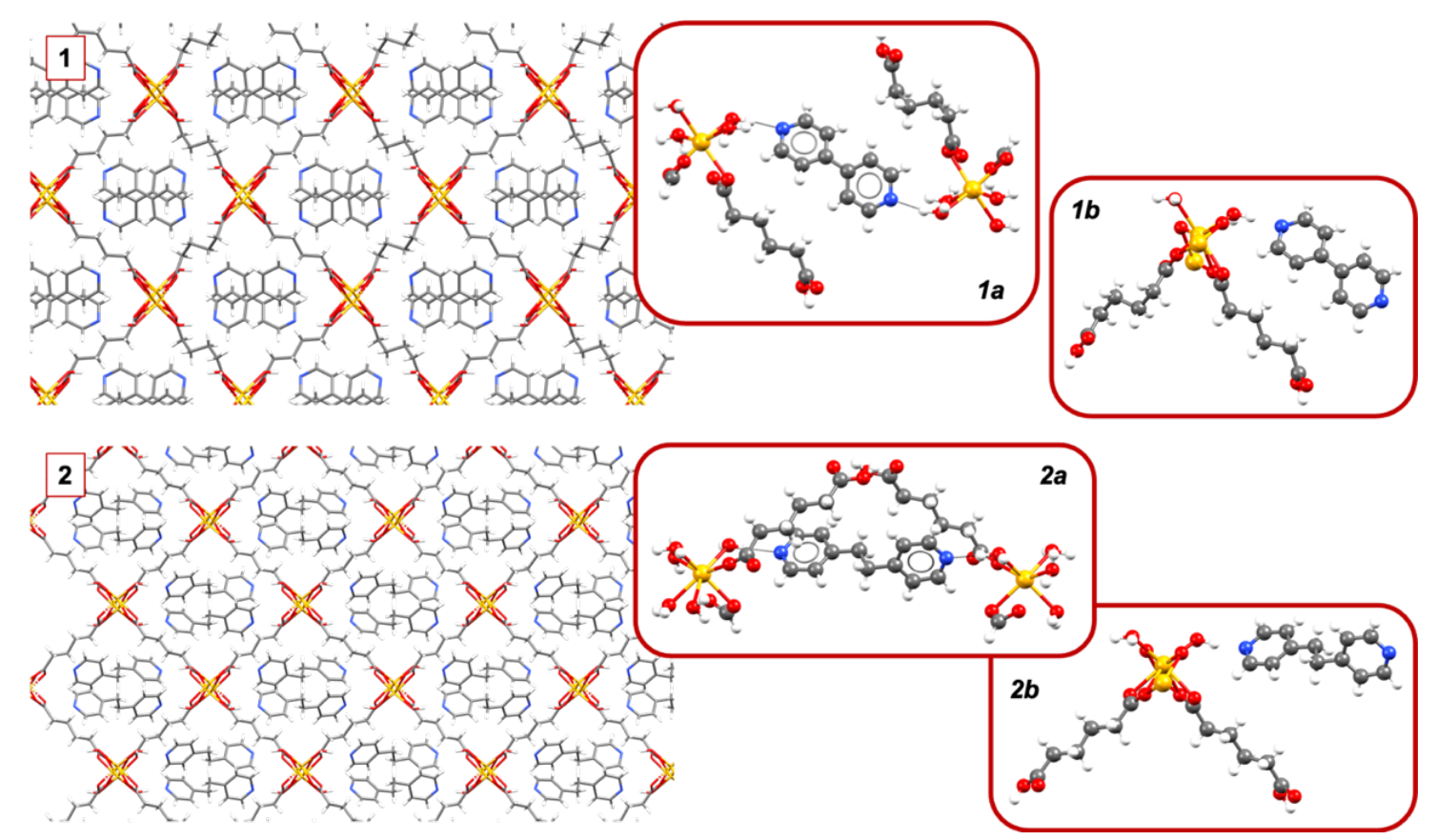

Figure 1. Crystal packing of systems 1 (4,4'-bipyridine guest) and 2 (1,2-bis(4-pyridyl)ethane guest), view in $c$ direction. Inlet: the chosen cluster models for each complex.

Finite models of the host-guest complexes, denoted here as 'cluster' models, were constructed from experimental geometries of $\mathbf{1}$ and $\mathbf{2}$ with the partially optimised positions of the coordinated water molecules. For each system, cluster models containing one guest molecule, hydrogen-bonded to the metal nodes of the MOF and the subsequent framework fragments of two sizes, $\mathbf{a}$ and $\mathbf{b}$, were considered (Figure 1). Terminal carboxylate groups of the adipates and unsaturated oxygen atoms of the metal node were hydrogenated for electric neutrality. Only the positions of these added hydrogen 
atoms were optimised, while the rest of the cluster was kept in the geometry from the periodic computations. Host-guest interaction energies (Eq. 2) in these clusters were computed using the same density functionals as in periodic computations in conjunction with a cc-pVTZ basis set and corrected for basis set superposition error (BSSE) using counterpoise correction: ${ }^{59}$

$\Delta E=E_{h g}-\left[E_{h}+E_{g}\right]+E^{B S S E}$

where $E_{h g}$ is the electronic energy of the supramolecular cluster, $E_{h}$ and $E_{g}$ are electronic energies of the truncated host and guest molecule in their cluster geometries, and $E^{B S S E}$ is the counterpoise correction. These computations were performed using Gaussian $16 .{ }^{60}$

Features of the electron density in the studied cluster models were analysed by means of QTAIM $^{57}$ (at the B3LYP/cc-pVTZ level of theory using AIMAII ${ }^{61}$ ), the non-covalent interactions $\left(\mathrm{NCI}^{62}\right.$ ) index (at the B3LYP/cc-pVTZ level, of theory using NCIplot ${ }^{63}$ ), and the density overlap regions indicator (DORI, ${ }^{64}$ at the B3LYP-D3/TZVP level of theory using ADF ${ }^{65}$ ). Furthermore, the total interaction energies in these complexes were analysed using two energy decomposition schemes: the energy decomposition analysis of Bickelhaupt and Baerends (BB-EDA, ${ }^{66}$ B3LYP-D3/TZVP level, using $\mathrm{ADF}^{65}$ ) and the symmetry-adapted perturbation theory (SAPT, ${ }^{67} \mathrm{SAPT} 0 / \mathrm{cc}-\mathrm{pVTZ}$ level, using Psi4 $\left.{ }^{68}\right)$.

\section{Results and Discussion}

\section{Periodic model}

Interaction energy. Interaction energies, computed as per Eq. 1 at a periodic DFT level with several functionals including the D3 dispersion correction, ${ }^{54}$ are given in Figure 2 and Table S1 of the ESI. MOF complex $\mathbf{2}$ is predicted to have stronger host-guest interactions relative to $\mathbf{1}$ with all considered methods; the gap between systems 1 and $\mathbf{2}$ is largest with LC- $\omega$ PBE-D3.

Electron density features. The topology of the electron density in the studied MOF complexes was analysed using QTAIM. ${ }^{57}$ Since the primary host-guest interactions in systems $\mathbf{1}$ and $\mathbf{2}$ are through hydrogen bonds, the properties of their bond critical points (BCPs) were examined (Table 1). Trends in these properties are in line with the hydrogen bond lengths: shorter contacts in system 2 are associated with higher density $\rho_{B C P}$ and Laplacian $\nabla^{2} \rho_{B C P}$, as well as more negative energy density $H_{B C P}$, relative to 1 . 


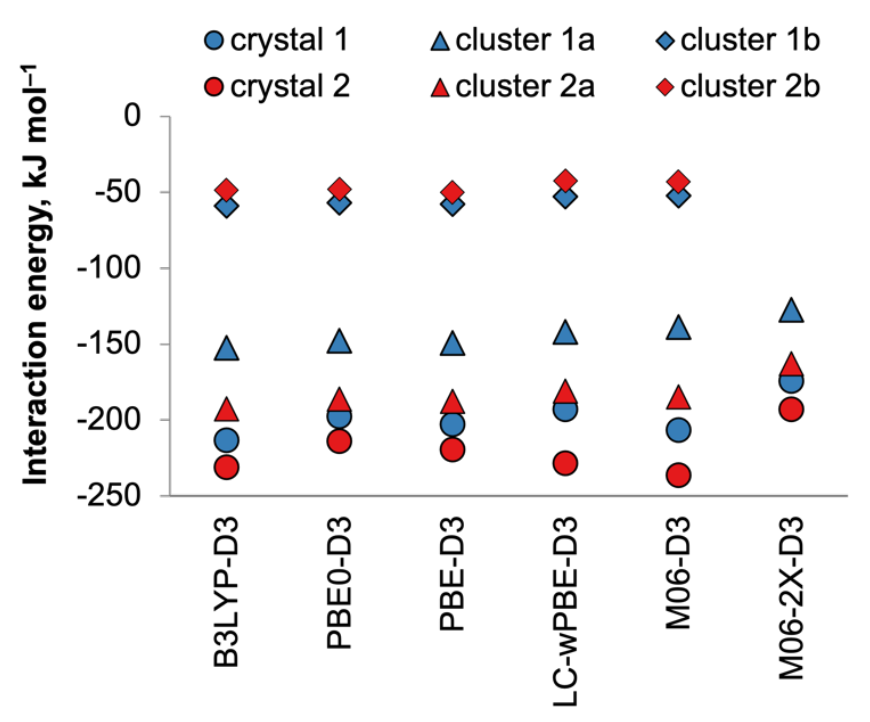

Figure 2. Host-guest interaction energies in periodic (Eq. 1; shown are interaction energies per guest molecule, i.e. $\Delta \mathrm{E} / 4$, as there are 4 guest molecules per unit cell) and cluster (Eq. 2) models of the studied systems, computed using a range of density functionals.

Table 1. QTAIM analysis of the hydrogen bonds in the studied host-guest complexes.

\begin{tabular}{|c|c|c|c|c|}
\hline System & $\begin{array}{l}\text { Bond length } \\
{[\AA]}\end{array}$ & $\begin{array}{l}\rho_{B C P}{ }^{a} \\
\text { [a.u.] }\end{array}$ & $\begin{array}{l}\nabla^{2} \rho_{B C P}{ }^{b} \\
\text { [a.u.] }\end{array}$ & $\begin{array}{l}H_{B C P}{ }^{c} \\
\text { [a.u.] }\end{array}$ \\
\hline \multicolumn{5}{|c|}{ Periodic model $^{d}$} \\
\hline \multirow[t]{2}{*}{1} & 1.883 & 0.035 & 0.090 & $-3.35 \mathrm{E}-03$ \\
\hline & 1.904 & 0.033 & 0.086 & $-2.87 \mathrm{E}-03$ \\
\hline \multirow[t]{2}{*}{2} & 1.806 & 0.042 & 0.097 & $-6.73 \mathrm{E}-03$ \\
\hline & 1.830 & 0.040 & 0.094 & $-5.80 \mathrm{E}-03$ \\
\hline \multicolumn{5}{|c|}{ Cluster model $\mathbf{a}^{d}$} \\
\hline \multirow[t]{2}{*}{1} & 1.883 & 0.036 & 0.068 & $-5.90 \mathrm{E}-03$ \\
\hline & 1.904 & 0.034 & 0.067 & $-5.11 \mathrm{E}-03$ \\
\hline \multirow[t]{2}{*}{2} & 1.806 & 0.044 & 0.064 & $-1.04 \mathrm{E}-02$ \\
\hline & 1.830 & 0.041 & 0.064 & $-9.14 \mathrm{E}-03$ \\
\hline \multicolumn{5}{|c|}{ Cluster model b } \\
\hline 1 & 1.883 & 0.035 & 0.071 & $-5.58 \mathrm{E}-03$ \\
\hline 2 & 1.806 & 0.043 & 0.072 & $-9.59 \mathrm{E}-03$ \\
\hline \multicolumn{5}{|c|}{$\mathrm{NH}_{3} \bullet \bullet \bullet \mathrm{H}_{2} \mathrm{O}$ cluster } \\
\hline $\mathrm{NH}_{3} \bullet \bullet \bullet \mathrm{H}_{2} \mathrm{O}$ & 1.700 & 0.055 & 0.083 & $-1.57 \mathrm{E}-02$ \\
\hline $\begin{array}{l}\mathrm{NH}_{3} \bullet \bullet \cdot \mathrm{H}_{2} \mathrm{O} \\
\text { (equilibrium) }\end{array}$ & 1.954 & 0.031 & 0.069 & $-3.00 \mathrm{E}-03$ \\
\hline $\mathrm{NH}_{3} \bullet \bullet \bullet \mathrm{H}_{2} \mathrm{O}$ & 2.200 & 0.018 & 0.049 & $8.56 \mathrm{E}-04$ \\
\hline
\end{tabular}




\section{Cluster model}

Interaction energy. Interaction energies in the supramolecular clusters (Eq. 2), obtained using several density functionals, are given in Figure 2 and Table S1 of the ESI. While there is no substantial variation between the results at different levels of theory, the relative interaction energies in the studied MOF-guest complexes are strongly influenced by the choice of the finite (cluster) model. The trend in $\Delta E$ for the cluster model a closely resembles that from the periodic computations. Qualitatively, complex 2 is more stabilised than complex 1; quantitatively, $\Delta E$ values for cluster model a are $30-70 \mathrm{~kJ} \mathrm{~mol}^{-1}$ above the interaction energies per guest molecule, computed for the periodic model. Cluster model $\mathbf{b}$ instead features much weaker (by as much as $c a .250 \mathrm{~kJ} \mathrm{~mol}^{-1}$ ) interaction energies, with system $\mathbf{1}$ being more stabilised than system $\mathbf{2}$.

To gain further insights into the nature of the host-guest interactions, decomposition analyses of the total interaction energies were performed. On one hand, within the energy decomposition framework of Bickelhaupt and Baerends (BB-EDA), ${ }^{66}$ the interaction energy consists of:

- the electrostatic interaction between the fragments with initial (frozen) charge density distributions;

- the exchange repulsion arising from Pauli's principle and corresponding to the destabilisation due to antisymmetrisation of the wavefunction;

- the orbital interaction energy comprising the charge transfer among fragments and the polarisation within fragments resulting from orbital mixing and orbital relaxation; and

- the additional dispersion term, e.g. the D3 correction.

On the other hand, weak (compared to covalent bonds) intermolecular interactions can be treated as perturbations to the wavefunctions of the individual molecules. This approach is embodied by the symmetry-adapted perturbation theory (SAPT), ${ }^{67}$ which provides access to accurate non-covalent interaction energies and their physically meaningful components:

- the electrostatic energy arising from the electrostatic interaction of the two monomer electron densities;

- the induction energy reflecting the response of one electron density to polarisation by the other electron density;

- the dispersion energy due to mutual polarisation of the two fragments by instantaneous fluctuations of their densities; and

- the exchange energy due to Pauli's exclusion principle. 
These two EDAs employ conceptually distinct approaches to quantifying the components of the total energy and thus allow for a comparative analysis of the studied systems. According to our results (Figure 3), the two schemes yield qualitatively similar pictures: the host-guest interaction in both $\mathbf{1}$ and 2 is primarily driven by electrostatics - a typical signature of hydrogen bonds, ${ }^{69}$ - although orbital (induction) and dispersion interactions are also significant. All energy components are notably smaller in cluster model $\mathbf{b}$ than in $\mathbf{a}$. Total interaction energies, computed with BB-EDA and SAPT0, are concordant with each other and with the DFT results.

Electron density features. The chosen QTAIM ${ }^{57}$ descriptors of the hydrogen bonds at their bond critical points in the supramolecular clusters are given in Table 1, and follow the same trends as in the periodic simulations. The electron densities at the BCPs in supramolecular clusters are almost identical to those obtained for the periodic model, while the Laplacian and energy density are only slightly lower.

(a) BB-EDA

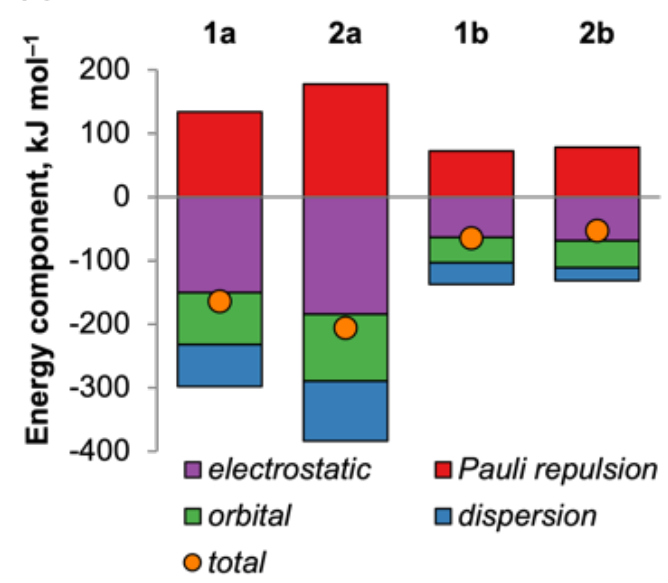

(b) SAPTO

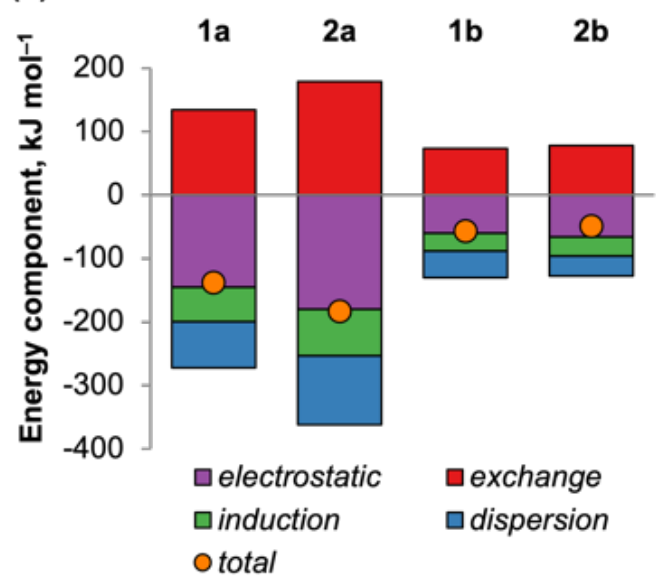

Figure 3. Energy decomposition analyses in cluster models a and $\mathbf{b}$ of MOF-guest complexes $\mathbf{1}$ and $\mathbf{2}$.

To put these results in perspective, we compared them with a prototypical hydrogen-bonded dimer, $\mathrm{NH}_{3} \bullet \bullet \mathrm{H}_{2} \mathrm{O}$, at three interaction distances (Table 1, see also Figure S4 in the ESI). As a consequence of their crystal packing, systems $\mathbf{1}$ and $\mathbf{2}$ feature shorter hydrogen bonds than that of the fully relaxed $\mathrm{NH}_{3} \bullet \bullet \mathrm{H}_{2} \mathrm{O}$. In general, the shorter the hydrogen bond, the higher the electron density and the Laplacian. ${ }^{70}$ The energy density at BCP is close to zero in all studied systems, as is typical for hydrogen bonds. These values are slightly negative, indicative of a somewhat predominated covalent character according to the criteria of Cremer and Kraka. ${ }^{71}$

The deformation density plots (Figure 4a) reveal two regions with an accumulation of electron density in the studied supramolecular clusters: $(i)$ the aforementioned hydrogen bonds between the MOF's water molecule and the guest's pyridinic nitrogen, and (ii) a less obvious interaction between the guest's $\mathrm{C}_{\mathrm{Ar}}-\mathrm{H}$ group and the oxygen atom of the adipate linker. 
The non-covalent interactions index ${ }^{62}$ exploits the features of electron density, its second derivative and reduced density gradient to analyse and visualise NCIs. A detailed discussion of the $\mathrm{NCI}$ analyses of the studied cluster models is available in the ESI, while computed isosurfaces of the reduced density gradient are shown in Figure $4 \mathrm{~b}$. Finally, supramolecular clusters were analysed by means of the density overlap regions indicator (DORI), ${ }^{64}$ which, unlike the NCI index, captures covalent and non-covalent interactions simultaneously (Figure 4c). Both the NCI index and DORI identify the same interaction patterns - pronounced hydrogen bonding between the framework's water molecule and the guest's pyridinic nitrogen, as well as additional interactions between the host's adipate backbone and the guest's $\mathrm{C}_{\mathrm{Ar}}-\mathrm{H}$ groups - all in clear agreement with the results of the deformation density analysis. Both tools also indicate, albeit on a qualitative level, that for cluster model $\mathbf{a}$, the interactions are more numerous and pronounced in complex $\mathbf{2}$ than in $\mathbf{1}$, while the opposite occurs in cluster model b.

\begin{tabular}{|c|c|c|c|}
\hline \multicolumn{2}{|c|}{ System 1} & \multicolumn{2}{|c|}{ System 2} \\
\hline cluster a & cluster $\mathbf{b}$ & cluster $\mathbf{a}$ & cluster $\mathbf{b}$ \\
\hline
\end{tabular}

\section{(a) Deformation density}
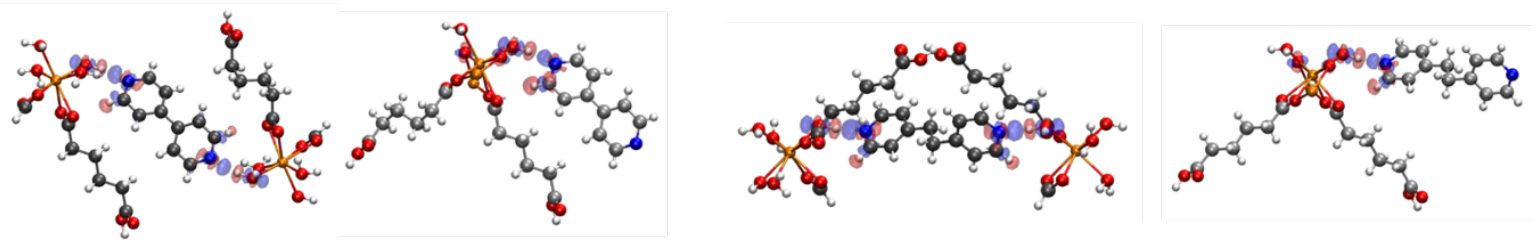

(b) $\mathrm{NCl}$ index
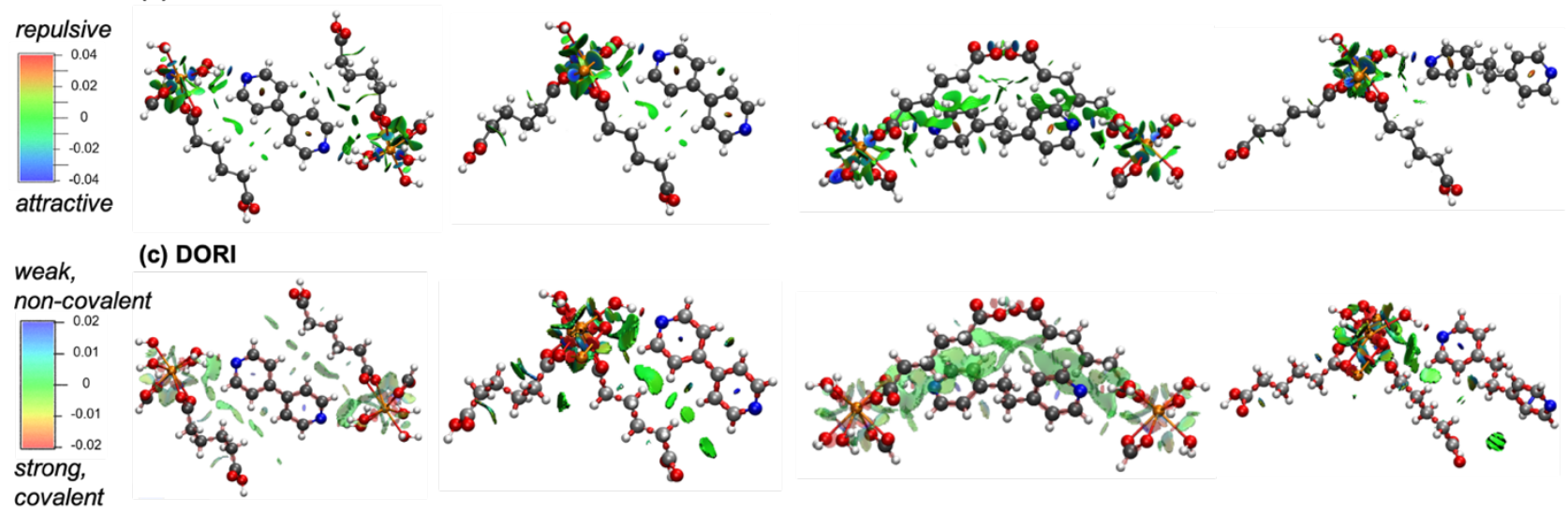

Figure 4. (a) Deformation density: full cluster density minus the sum of the fragment densities (blue and red represent positive and negative values, respectively; the surface isovalue is 0.0015 a.u.). (b) NCI index surfaces corresponding to $\mathrm{s}=0.5$ a.u. and the colour scale -0.04 a.u. (blue) $<\rho<0.04$ a.u. (red). (c) DORI surfaces at 0.98 isovalue, colour-coded by $\operatorname{sign}\left(\lambda_{2}\right) \rho(r)$ in the range from -0.02 a.u. (red) to 0.02 a.u. (blue). Note the opposite colour schemes for NCI and DORI surfaces, chosen in accordance with the literature standards. 


\section{Discussion}

The choice of a finite model for a periodic system can have important effects on its qualitative description. We demonstrate this by comparing the computed interaction energies for cluster models $\mathbf{a}$ and $\mathbf{b}$ to those for the periodic model, as well as to the indirect experimental evidence. Specifically, thermogravimetric analysis in the original study of PARHAS and PARHEW MOFs ${ }^{53}$ suggests that complex 2 features somewhat stronger interactions between MOF and guest than complex $\mathbf{1}$. This is reflected in the interaction energies, computed for the periodic model and cluster model a, but not cluster model b. The latter therefore does not provide adequate representation of the host-guest interactions. In light of the scarcity of accurate structural data for other MOF-guest complexes, our results stress the need for sufficiently large models in order to have any interpretative or predictive value in this domain.

Provided an appropriate finite model is chosen, it can be analysed with a plethora of quantumchemical tools to obtain valuable insights into the nature of the interactions between the host framework and its molecular guest. First, direct comparison of the QTAIM results between the periodic and cluster models reveals their strong quantitative agreement, further validating the ability of a suitable finite model to describe the non-covalent interactions in the real system. In the case of the species studied herein, the primary host-guest interaction, i.e., the hydrogen bond between the guest's pyridinic nitrogen and the MOF's water molecule, is shorter and consequently stronger in system 2 compared to 1 (Table 1). The NCI index and DORI results for the supramolecular clusters (Figure 4) confirm the predominant role of these hydrogen bonds in the interaction between MOF and guest. Thus, the 'obvious' hydrogen bonds are readily captured by all the tools employed in both the periodic and finite models. Furthermore, NCI and DORI indicate that other weakly stabilising interactions between the guest and the framework occur and are more abundant in system $\mathbf{2}$ than in 1. These results highlight the need for both local, such as QTAIM, and global, e.g., NCI and DORI, bonding analyses to fully capture the relevant non-covalent interactions.

Finally, all energy components are larger in complex 2 than complex 1 with both the BickelhauptBaerends and SAPT energy decomposition analyses (Figure 3). While both systems feature a significant stabilising electrostatic component corresponding to hydrogen bonding, the most pronounced difference between them arises from dispersion. This further emphasises the crucial role of secondary host-guest interactions. 


\section{Conclusions}

We investigated the strength and nature of non-covalent interactions between small drug molecules and a metal-organic framework host with a range of computational chemistry tools using periodic and finite (cluster) models. On the basis of the results obtained here, the following methodological implications for future in silico modelling of these systems arise. First, the choice of a supramolecular cluster representing the MOF-molecule complex is crucial for computing qualitatively correct interaction energies and analysing the non-covalent interactions responsible for them. In this work, two types of cluster model produced contrasting results, with the larger model accurately reproducing the data from periodic computations. Importantly, DFT and wavefunctiontheory computations are still practicable for the larger clusters studied herein. Second, all tested dispersion-corrected density functionals, including general gradient approximation (GGA), hybrid, meta-GGA, and long-range corrected methods, yield qualitatively similar results for interaction energies with both infinite and cluster models. These DFT results are also in agreement with the total interaction energies obtained using two energy decomposition schemes, the Bickelhaupt-Baerends EDA and the symmetry-adapted perturbation theory. Despite being conceptually different in their treatment of various energy components, these EDA schemes yield a very similar qualitative picture for the studied MOF complexes. Comparing their performance against the periodic energy decomposition analyses ${ }^{72}$ could be an ultimate validation; however, such tools have not yet been adapted specifically to MOFs. Third, excellent agreement between the results of Bader's quantum theory of atoms in molecules for crystal and cluster models supports the transferability of the qualitative trends from finite to periodic systems, provided the former are chosen appropriately. However, the QTAIM analysis itself is only suitable for studying the specific, pre-selected interactions. A more generalised, albeit rather qualitative, picture of the non-covalent interactions in MOFs can be achieved with the help of the non-covalent interactions index and the density overlap regions indicator. The latter two approaches enable the detection and visualisation of various intermolecular interactions, as well as qualitative assignment of their nature, e.g., attractive vs. repulsive, and strong vs. weak. DORI has an added benefit of capturing the inter- and intramolecular interactions simultaneously.

A deeper understanding of the underlying interactions in MOF-guest complexes, afforded by the quantum-chemical tools tested here, can enhance the rational design of MOFs toward target applications. In this work, we have demonstrated that tools such as QTAIM, NCI index, and DORI, commonplace in the domain of small molecule modelling, have immense utility when transferred to organic frameworks. Many such tools are currently practical only for cluster models; however, the 
latter, when constructed carefully, yield reliable energies and types of non-covalent interactions when compared to periodic results.

\section{Author Contributions}

Michelle Ernst: conceptualisation; methodology; investigation; formal analysis; visualisation; writing - original draft, review and editing. Ganna Gryn'ova: conceptualisation; methodology; formal analysis; visualisation; writing - review and editing; supervision.

\section{Conflicts of interest}

There are no conflicts to declare.

\section{Acknowledgements}

The authors acknowledge support from the Klaus Tschira Foundation. M.E acknowledges support from the Swiss National Science Foundation (project number: P2BEP2_195236). The authors thank the state of Baden-Württemberg through bwHPC (bwForCluster JUSTUS2) and the University of Bern (HPC cluster UBELIX, http://www.id.unibe.ch/hpc) for granting access to computational resources. The authors thank Dr John Lindner for proof-reading the manuscript.

\section{Notes}

I The basis set superposition error in the periodic computations, assessed using the geometrical counterpoise correction, does not affect the qualitative trends; see further details in the ESI.

$\S$ While the Dunning basis sets augmented with diffuse functions are recommended for SAPT computations, these are unfortunately not available for calcium in Psi4.

\section{References}

\footnotetext{
${ }^{1}$ B. F. Hoskins and R. Robson, Design and construction of a new class of scaffolding-like materials comprising infinite polymeric frameworks of 3D-linked molecular rods. A reappraisal of the zinc cyanide and cadmium cyanide structures and the synthesis and structure of the diamond-related frameworks $[\mathrm{N}(\mathrm{CH} 3) 4][\mathrm{CuIZnII}(\mathrm{CN}) 4]$ and $\mathrm{CuI}[4,4$ ',4",4"'-tetracyanotetraphenylmethane]BF4.xC6H5NO2. J. Am. Chem. Soc., 1990, 112, 1546-1554, DOI: 10.1021/ja00160a038.
} 
${ }^{2}$ O. M. Yaghi and H. Li, Hydrothermal Synthesis of a Metal-Organic Framework Containing Large Rectangular Channels. J. Am. Chem. Soc. 1995, 117, 10401-10402, DOI: 10.1021/ja00146a033

${ }^{3}$ B. Li, H.-M. Wen, W. Zhou and B. Chen, Porous Metal-Organic Frameworks for Gas Storage and Separation: What, How, and Why? J. Phys. Chem. Lett., 2014, 5, 3468-3479, DOI: 10.1021/jz501586e.

${ }^{4}$ H. Li, K. Wang, Y. Sun, C. T. Lollar, J. Li and H.-C. Zhou, Recent Advances in Gas Storage and Separation Using Metal-Organic Frameworks. Mater. Today, 2018, 21, 108-121, DOI: 10.1016/j.mattod.2017.07.006.

${ }^{5}$ P. Horcajada, C. Serre, M. Vallet-Regí, M. Sebban, F. Taulelle and G. Férey, Metal-Organic Frameworks as Efficient Materials for Drug Delivery. Angew. Chem. Int. Ed., 2006, 45, 5974-5978, DOI: 10.1002/anie.200601878.

${ }^{6}$ F. Llabres i Xamena, A. Abad, A. Corma and H. Garcia, MOFs as Catalysts: Activity, Reusability and ShapeSelectivity of a Pd-Containing MOF. J. Catal., 2007, 250, 294-298, DOI: 10.1016/j.jcat.2007.06.004.

${ }^{7}$ U. Ravon, M. E. Domine, C. Gaudillère, A. Desmartin-Chomel and D. Farrusseng, MOFs as Acid Catalysts with Shape Selectivity Properties. New J. Chem., 2008, 32, 937-940, DOI: 10.1039/B803953B.

${ }^{8}$ P. F. Muldoon, G. Collet, S. V. Eliseeva, T.-Y. Luo, S. Petoud, and N. L. Rosi, Ship-in-a-Bottle Preparation of Long Wavelength Molecular Antennae in Lanthanide Metal-Organic Frameworks for Biological Imaging, J. Am. Chem. Soc., 2020, 142, 8776-8781, DOI: 10.1021/jacs.0c01426.

${ }^{9}$ A. Foucault-Collet, K. A. Gogick, K. A. White, S. Villette, AA.gnès Pallier, G. Collet, C. Kieda, T. Li, S. J. Geib, N. L. Rosi and S. Petoud, Lanthanide near infrared imaging in living cells with $\mathrm{Yb}^{3+}$ nano metal organic frameworks. Proc. Natl. Acad. Sci. USA, 2013, 110, 17199-17204; DOI: 10.1073/pnas.1305910110.

${ }^{10}$ S. Zhang, X. Pei, H. Gao, S. Chen and J.Wang, Metal-organic framework-based nanomaterials for biomedical applications. Chin. Chem. Lett., 2020, 31, 1060-1070, DOI: 10.1016/j.cclet.2019.11.036.

${ }^{11}$ R. B. Getman, Y.-S. Bae, C. E. Wilmer, R. Q. Snurr, Review and Analysis of Molecular Simulations of Methane, Hydrogen, and Acetylene Storage in Metal-Organic Frameworks. Chem. Rev., 2012, 112, 703-723, DOI: $10.1021 / \mathrm{cr} 200217 \mathrm{c}$.

${ }^{12}$ Y.-Z. Li, G.-D. Wang, H-Y. Yang, L. Hou, Y.-Y. Wang and Z. Zhu, Novel cage-like MOF for gas separation, CO2 conversion and selective adsorption of an organic dye. Inorg. Chem. Front., 2020, 7, 746-755, DOI: 10.1039/C9QI01262A.

${ }^{13}$ D.-Y. Ma, Z. Li, J.-X. Xiao, R. Deng, P.-F. Lin, R.-Q. Chen, Y.-Q. Liang, H.-F. Guo, B. Liu and J.-Q. Liu, Hydrostable and Nitryl/Methyl-Functionalized Metal-Organic Framework for Drug Delivery and Highly Selective CO2 Adsorption. Inorg. Chem. 2015, 54, 6719-6726, DOI: 10.1021/acs.inorgchem.5b00335.

${ }^{14}$ V. Bernales, M. A. Ortuño, D. G. Truhlar, C. J. Cramer and L. Gagliardi, Computational Design of Functionalized Metal-Organic Framework Nodes for Catalysis. ACS Cent. Sci. 2018, 4, 5-19, DOI: 10.1021/acscentsci.7b00500. 
${ }^{15}$ M. C. Bernini, D. Fairen-Jimenez, M. Pasinetti, A. J. Ramirez-Pastor and R. Q. Snurr, Screening of biocompatible metal-organic frameworks as potential drug carriers using Monte Carlo simulations. J. Mater. Chem. B, 2014, 2, 766-774, DOI: 10.1039/C3TB21328E.

${ }^{16}$ R. Babarao and J. Jiang, Unraveling the Energetics and Dynamics of Ibuprofen in Mesoporous Metal-Organic Frameworks. J. Phys. Chem. C, 2009, 113, 18287-18291, DOI: 10.1021/jp906429s.

${ }^{17}$ M. Kotzabasaki and G. E. Froudakis, Review of computer simulations on anti-cancer drug delivery in MOFs. Inorg. Chem. Front., 2018, 5, 1255-1272, DOI: 10.1039/C7QI00645D.

${ }^{18}$ T. Mueller and G. Ceder, A Density Functional Theory Study of Hydrogen Adsorption in MOF-5. J. Phys. Chem. B, 2005, 109, 17974-17983, DOI: 10.1021/jp051202q.

${ }^{19}$ M. Delle Piane, M. Corno, A. Pedone, R. Dovesi, and P. Ugliengo, Large-Scale B3LYP Simulations of Ibuprofen Adsorbed in MCM-41 Mesoporous Silica as Drug Delivery System. J. Phys. Chem. C 2014, 118, 26737-26749, DOI: 10.1021/jp507364h.

${ }^{20}$ R. Scatena, Y. Guntern, and P. Macchi, Electron density and dielectric properties of highly porous MOFs: binding and mobility of guest molecules in Cu3 (BTC) 2 and Zn3 (BTC) 2." J. Am. Chem. Soc, 2019, 141, 9382-9390.

${ }^{21}$ T. Watanabe, S. Keskin, S. Nair and D. S. Sholl, Computational identification of a metal organic framework for high selectivity membrane-based $\mathrm{CO} 2 / \mathrm{CH} 4$ separations: $\mathrm{Cu}$ (hfipbb)(H2hfipbb)0.5. Phys. Chem. Chem. Phys., 2009, 11, 11389-11394, DOI: 10.1039/B918254N.

${ }^{22}$ L. Grajciar, O. Bludský and P. Nachtigall, Water Adsorption on Coordinatively Unsaturated Sites in CuBTC MOF. J. Phys. Chem. Lett., 2010, 1, 3354-3359, DOI: 10.1021/jz101378z.

${ }^{23}$ Y.-H. Kim, J. Kang and S.-H. Wei, Origin of Enhanced Dihydrogen-Metal Interaction in Carboxylate Bridged Cu2-Paddle-Wheel Frameworks. Phys. Rev. Lett., 2010, 105, 236105, DOI: 10.1103/PhysRevLett.105.236105.

${ }^{24}$ K. Sillar, A. Hofmann and J. Sauer, Ab Initio Study of Hydrogen Adsorption in MOF-5. J. Am. Chem. Soc., 2009, 131, 4143-4150, DOI: 10.1021/ja8099079.

${ }^{25}$ K. Sillar, J. Sauer, Ab Initio Prediction of Adsorption Isotherms for Small Molecules in Metal-Organic Frameworks: The Effect of Lateral Interactions for Methane/CPO-27-Mg, J. Am. Chem. Soc., 2012, 134, 18354-18365, DOI: 10.1021/ja307076t.

${ }^{26}$ M. Dixit,T. A. Maark, S. Pal Ab initio and periodic DFT investigation of hydrogen storage on light metaldecorated MOF-5. Int. J. Hydrog. Energy, 2011, 36, 10816-10827, DOI: 10.1016/j.ijhydene.2011.05.165.

${ }^{27}$ R. Poloni, B. Smit and J. B. Neaton, Ligand-Assisted Enhancement of CO2 Capture in Metal-Organic Frameworks. J. Am. Chem. Soc., 2012, 134, 6714-6719, DOI: 10.1021/ja2118943.

${ }^{28}$ H. Kim and Y. Jung, Can Metal-Organic Framework Separate 1-Butene from Butene Isomers? J. Phys. Chem. Lett., 2014, 5, 440-446, DOI: 10.1021/jz402734x. 
${ }^{29}$ L. C. Gallington, I. S. Kim, W.-G. Liu, A. A. Yakovenko, A. E. Platero-Prats, Z. Li, T. C. Wang, J. T. Hupp, O. K. Farha, D. G. Truhlar, A. B. F. Martinson and K. W. Chapman, Regioselective Atomic Layer Deposition in Metal-Organic Frameworks Directed by Dispersion Interactions. J. Am. Chem. Soc. 2016, 138, 1351313516, DOI: 10.1021/jacs.6b08711.

${ }^{30}$ P. Horcajada, C. Serre, G. Maurin, N. A. Ramsahye, F. Balas, M. Vallet-Regí, M. Sebban, F. Taulelle and G. Férey, Flexible Porous Metal-Organic Frameworks for a Controlled Drug Delivery. J. Am. Chem. Soc., 2008, 130, 6774-6780, DOI: 10.1021/ja710973k.

${ }^{31}$ C. Vieira Soares, G. Maurin and A. A. Leitão, Computational Exploration of the Catalytic Degradation of Sarin and Its Simulants by a Titanium Metal-Organic Framework. J. Phys. Chem. C, 2019, 123, 19077-19086, DOI: $10.1021 /$ acs.jpcc.9b05838.

${ }^{32}$ E. N. Koukaras, T. Montagnon, P. Trikalitis, D. Bikiaris\|, A. D. Zdetsis and G. E. Froudakis, Toward Efficient Drug Delivery through Suitably Prepared Metal-Organic Frameworks: A First-Principles Study. J. Phys. Chem. C, 2014, 118, 8885-8890, DOI: 10.1021/jp410282m.

${ }^{33}$ M. Kotzabasaki, I. Galdadas, E. Tylianakis, E. Klontzas, Z. Cournia and G. E. Froudakis, Multiscale simulations reveal IRMOF-74-III as a potent drug carrier for gemcitabine delivery. J. Mater. Chem. B, 2017, 5, 3277-3282, DOI: 10.1039/C7TB00220C.

${ }^{34}$ Y. Yao, X. Song, J. Qiu and C. Hao, Interaction between Formaldehyde and Luminescent MOF $[\mathrm{Zn}(\mathrm{NH} 2 \mathrm{bdc})(\mathrm{bix})] \mathrm{n}$ in the Electronic Excited State. J. Phys. Chem. A, 2014, 118, 6191-6196, DOI: $10.1021 /$ jp503722m.

${ }^{35}$ E. Pastorczak and C. Corminboeuf, Found in Translation: Quantum Chemical Tools for Grasping NonCovalent Interactions. J. Chem. Phys., 2017, 146, 120901, DOI: 10.1063/1.4978951.

${ }^{36}$ J. L. Mancuso, A. M. Mroz, K. N. Le and C. H. Hendon, Electronic Structure Modeling of Metal-Organic Frameworks. Chem. Rev., 2020, 120, 8641-8715, DOI: 10.1021/acs.chemrev.0c00148.

${ }^{37}$ M. Busch, R. B. Wang, A. Hellman, J. Rossmeisl and H. Grönbeck, The Influence of Inert Ions on the Reactivity of Manganese Oxides. J. Phys. Chem. C, 2018, 122, 216-226, DOI: 10.1021/acs.jpcc.7b10760.

${ }^{38}$ I. Casademont-Reig, T. Woller, J. Contreras-García, M. Alonso, M. Torrent-Sucarrat and E. Matito, New electron delocalization tools to describe the aromaticity in porphyrinoids. Phys. Chem. Chem. Phys., 2018, 20, 2787-2796, DOI: 10.1039/C7CP07581B.

${ }^{39}$ L. J. Karas, A. T. Campbell, I. V. Alabugin, and J. I. Wu, Antiaromaticity Gain Activates Tropone and Nonbenzenoid Aromatics as Normal-Electron-Demand Diels-Alder Dienes. Org. Lett., 2020, 22, 7083-7087, DOI: 10.1021/acs.orglett.0c02343.

${ }^{40}$ I. Fernández and G. Frenking, The Diels-Alder Reaction from the EDA-NOCV Perspective: A ReExamination of the Frontier Molecular Orbital Model. Eur. J. Org. Chem., 2019, 478-485, DOI: 10.1002/ejoc.201800888. 
${ }^{41}$ R. Lee, G. Gryn'ova, K. U. Ingold and M. L. Coote, Why are sec-alkylperoxyl bimolecular self-reactions orders of magnitude faster than the analogous reactions of tert-alkylperoxyls? The unanticipated role of $\mathrm{CH}$ hydrogen bond donation. Phys. Chem. Chem. Phys., 2016, 18, 23673-23679, DOI: 10.1039/C6CP04670C.

${ }^{42}$ B. W. Bakra and C. D. Sherrill, Analysis of transition state stabilization by non-covalent interactions in organocatalysis: application of atomic and functional-group partitioned symmetry-adapted perturbation theory to the addition of organoboron reagents to fluoroketones. Phys. Chem. Chem. Phys., 2018, 20, 18241-18251, DOI: $10.1039 /$ C8CP02029A.

${ }^{43}$ R. M. H. Ugale and S. E. Wheeler, Understanding the Reactivity and Selectivity of Fluxional Chiral DMAPCatalyzed Kinetic Resolutions of Axially Chiral Biaryls. Chem. Eur. J., 2019, 25, 4452-4459, DOI: 10.1002/chem.201806068.

${ }^{44}$ P. Vermeeren, T. A. Hamlin, F. M. Bickelhaupt and I. Fernández, Bifunctional Hydrogen Bond DonorCatalyzed Diels-Alder Reactions: Origin of Stereoselectivity and Rate Enhancement. Chem. Eur. J., 2020, Early View, DOI: 10.1002/chem.202004496.

${ }^{45}$ M. J. S. Phipps, T. Fox, C. S. Tautermann and C.-K. Skylaris, Energy decomposition analysis approaches and their evaluation on prototypical protein-drug interaction patterns. Chem. Soc. Rev., 2015, 44, 3177-3211, DOI: $10.1039 / \mathrm{C} 4 \mathrm{CS} 00375 \mathrm{~F}$.

${ }^{46}$ D. Arias-Olivares, E. K. Wieduwilt, J. Contreras-García, and A. Genoni, NCI-ELMO: A New Method To Quickly and Accurately Detect Noncovalent Interactions in Biosystems. J. Chem. Theory Comput., 2019, 15, 6456-6470, DOI: 10.1021/acs.jctc.9b00658.

${ }^{47}$ B. Meyer, S. Barthel, A. Mace, L. Vannay, B. Guillot, B. Smit, and C. Corminboeuf, DORI Reveals the Influence of Noncovalent Interactions on Covalent Bonding Patterns in Molecular Crystals Under Pressure. J. Phys. Chem. Lett., 2019, 10, 1482-1488, DOI: 10.1021/acs.jpclett.9b00220.

${ }^{48}$ N. Casati, A. Kleppe, A. Jephcoat and P. Macchi, Putting pressure on aromaticity along with in situ experimental electron density of a molecular crystal. Nat. Commun., 2016, 7, 10901, DOI: $10.1038 /$ ncomms 10901 .

${ }^{49}$ C. Foroutan-Nejad, M. Straka. I. Fernández and G. Frenking, Buckyball Difluoride F2-@C60+-A SingleMolecule Crystal. Angew. Chem. Int. Ed., 2018, 130, 14127-14130, DOI: 10.1002/ange.201809699.

${ }^{50}$ P. R. Varadwaj, A. Varadwaj, H. M. Marques and K. Yamashita, Significance of hydrogen bonding and other noncovalent interactions in determining octahedral tilting in the $\mathrm{CH} 3 \mathrm{NH} 3 \mathrm{PbI} 3$ hybrid organic-inorganic halide perovskite solar cell semiconductor. Sci. Rep., 2019, 9, 50, DOI: 10.1038/s41598-018-36218-1.

${ }^{51}$ Z. Wen and J. I.-C. Wu, Antiaromaticity gain increases the potential for n-type charge transport in hydrogenbonded $\pi$-conjugated cores. Chem. Commun., 2020, 56, 2008-2011, DOI: 10.1039/C9CC09670A.

${ }^{52}$ G. Gryn'ova and C. Corminboeuf, Implications of Charge Penetration for Heteroatom-Containing Organic Semiconductors. J. Phys. Chem. Lett., 2016, 7, 5198-5204, DOI: 10.1021/acs.jpclett.6b02585. 
${ }^{53}$ D. T. de Lill, D. J. Bozzuto and C. L. Cahill, Templated metal-organic frameworks: synthesis, structures, thermal properties and solid-state transformation of two novel calcium-adipate frameworks. Dalton Trans., 2005, 2111-2115, DOI: 10.1039/B502512E.

${ }^{54}$ J. Moellmann and S. Grimme, DFT-D3 Study of Some Molecular Crystals. J. Phys. Chem. C, 2014, 118, 7615-7621, DOI: 10.1021/jp501237c.

${ }^{55}$ D. Vilela Oliveira, M. F. Peintinger, J. Laun, and T. Bredow, B BSSE-correction scheme for consistent gaussian basis sets of double- and triple-zeta valence with polarization quality for solid-state calculations. J. Comp. Chem., 2019, 40, 2364-2376, DOI: 10.1002/jcc.26013

${ }^{56}$ R. Dovesi, A. Erba, R. Orlando, C. M. Zicovich-Wilson, B. Civalleri, L. Maschio, M. Rerat, S. Casassa, J. Baima, S. Salustro and B. Kirtman, Quantum-mechanical condensed matter simulations with CRYSTAL. WIREs Comput Mol Sci., 2018, 8, e1360, DOI: 10.1002/wcms.1360; R. Dovesi, V. R. Saunders, C. Roetti, R. Orlando, C. M. Zicovich-Wilson, F. Pascale, B. Civalleri, K. Doll, N. M. Harrison, I. J. Bush, P. D’Arco, M. Llunell, M. Causà, Y. Noël, L. Maschio, A. Erba, M. Rerat and S. Casassa, CRYSTAL17, (2017) CRYSTAL17 User's Manual. University of Torino, Torino.

${ }^{57}$ R. F. W. Bader, Atoms in molecules. Acc. Chem. Res., 1985, 18, 9-15, DOI: 10.1021/ar00109a003.

${ }^{58}$ C. Gatti, V. R. Saunders and C. Roetti, Crystal field effects on the topological properties of the electron density in molecular crystals: The case of urea. J. Chem. Phys., 1994, 101, 10686, DOI: 10.1063/1.467882.

${ }^{59}$ S. Simon, M. Duran and J. J. Dannenberg, How does basis set superposition error change the potential surfaces for hydrogen bonded dimers? J. Chem. Phys., 1996, 105, 11024-31, DOI: 10.1063/1.472902.

${ }^{60}$ Gaussian 16, Revision C.01, M. J. Frisch, G. W. Trucks, H. B. Schlegel, G. E. Scuseria, M. A. Robb, J. R. Cheeseman, G. Scalmani, V. Barone, G. A. Petersson, H. Nakatsuji, X. Li, M. Caricato, A. V. Marenich, J. Bloino, B. G. Janesko, R. Gomperts, B. Mennucci, H. P. Hratchian, J. V. Ortiz, A. F. Izmaylov, J. L. Sonnenberg, D. Williams-Young, F. Ding, F. Lipparini, F. Egidi, J. Goings, B. Peng, A. Petrone, T. Henderson, D. Ranasinghe, V. G. Zakrzewski, J. Gao, N. Rega, G. Zheng, W. Liang, M. Hada, M. Ehara, K. Toyota, R. Fukuda, J. Hasegawa, M. Ishida, T. Nakajima, Y. Honda, O. Kitao, H. Nakai, T. Vreven, K. Throssell, J. A. Montgomery, Jr., J. E. Peralta, F. Ogliaro, M. J. Bearpark, J. J. Heyd, E. N. Brothers, K. N. Kudin, V. N. Staroverov, T. A. Keith, R. Kobayashi, J. Normand, K. Raghavachari, A. P. Rendell, J. C. Burant, S. S. Iyengar, J. Tomasi, M. Cossi, J. M. Millam, M. Klene, C. Adamo, R. Cammi, J. W. Ochterski, R. L. Martin, K. Morokuma, O. Farkas, J. B. Foresman, and D. J. Fox, Gaussian, Inc., Wallingford CT, 2016.

${ }^{61}$ AIMAll (Version 19.10.12), T. A. Keith and T. K. Gristmill Software, Overland Park KS, USA, 2019 (aim.tkgristmill.com).

${ }^{62}$ E. R. Johnson, S. Keinan, P. Mori-Sánchez, J. Contreras-García, A. J. Cohen and W. Yang, Revealing Noncovalent Interactions. J. Am. Chem. Soc., 2010, 132, 6498-6506, DOI: 10.1021/ja100936w. 
${ }^{63}$ J. Contreras-García, E. R. Johnson, S. Keinan, R. Chaudret, J.-P. Piquemal, D. N. Beratan and W. Yang, NCIPLOT: A Program for Plotting Noncovalent Interaction Regions. J. Chem. Theory Comput., 2011, 7, 625632, DOI: $10.1021 / \mathrm{ct100641a.}$

${ }^{64}$ P. de Silva and C. Corminboeuf, Simultaneous Visualization of Covalent and Noncovalent Interactions Using Regions of Density Overlap. J. Chem. Theory Comput., 2014, 10, 3745-3756, DOI: 10.1021/ct500490b. ${ }^{65}$ G. te Velde, F. M. Bickelhaupt, E. J. Baerends, C. Fonseca Guerra, S. J. A. van Gisbergen, J. G. Snijders and T. Ziegler, Chemistry with ADF. J. Comput. Chem., 2001, 22, 931-967, DOI: 1 0.1002/jcc.1056; ADF 2019.304, SCM, Theoretical Chemistry, Vrije Universiteit, Amsterdam, The Netherlands, http://www.scm.com. Optionally, you may add the following list of authors and contributors: E. J. Baerends, T. Ziegler, A. J. Atkins, J. Autschbach, O. Baseggio, D. Bashford, A. Bérces, F. M. Bickelhaupt, C. Bo, P. M. Boerrigter, L. Cavallo, C. Daul, D. P. Chong, D. V. Chulhai, L. Deng, R. M. Dickson, J. M. Dieterich, D. E. Ellis, M. van Faassen, L. Fan, T. H. Fischer, A. Förster, C. Fonseca Guerra, M. Franchini, A. Ghysels, A. Giammona, S. J. A. van Gisbergen, A. Goez, A. W. Götz, J. A. Groeneveld, O. V. Gritsenko, M. Grüning, S. Gusarov, F. E. Harris, P. van den Hoek, Z. Hu, C. R. Jacob, H. Jacobsen, L. Jensen, L. Joubert, J. W. Kaminski, G. van Kessel, C. König, F. Kootstra, A. Kovalenko, M. V. Krykunov, E. van Lenthe, D. A. McCormack, A. Michalak, M. Mitoraj, S. M. Morton, J. Neugebauer, V. P. Nicu, L. Noodleman, V. P. Osinga, S. Patchkovskii, M. Pavanello, C.A. Peeples, P. H. T. Philipsen, D. Post, C. C. Pye, H. Ramanantoanina, P. Ramos, W. Ravenek, M. Reimann, J. I. Rodríguez, P. Ros, R. Rüger, P. R. T. Schipper, D. Schlüns, H. van Schoot, G. Schreckenbach, J. S. Seldenthuis, M. Seth, J. G. Snijders, M. Solà, M. Stener, M. Swart, D. Swerhone, V. Tognetti, G. te Velde, P. Vernooijs, L. Versluis, L. Visscher, O. Visser, F. Wang, T. A. Wesolowski, E. M. van Wezenbeek, G. Wiesenekker, S. K. Wolff, T. K. Woo, A. L. Yakovlev.

${ }^{66}$ F. M. Bickelhaupt and E. J. Baerends, Kohn-Sham Density Functional Theory: Predicting and Understanding Chemistry, in Rev. Comput. Chem., ed. K. B. Lipkowitz and D. B. Boyd, Wiley-VCH, New York, 2000, Vol. 15, 1-86.

${ }^{67}$ B. Jeziorski, R. Moszynski, K. Szalewicz, Perturbation Theory Approach to Intermolecular Potential Energy Surfaces of van der Waals Complexes. Chem. Rev., 1994, 94, 1887-1930, DOI: 10.1021/cr00031a008.

${ }^{68}$ Psi4 1.3.2: An Open-Source Electronic Structure Program Emphasizing Automation, Advanced Libraries, and Interoperability", R. M. Parrish, L. A. Burns, D. G. A. Smith, A. C. Simmonett, A. E. DePrince III, E. G. Hohenstein, U. Bozkaya, A. Yu. Sokolov, R. Di Remigio, R. M. Richard, J. F. Gonthier, A. M. James, H. R. McAlexander, A. Kumar, M. Saitow, X. Wang, B. P. Pritchard, P. Verma, H. F. Schaefer III, K. Patkowski, R. A. King, E. F. Valeev, F. A. Evangelista, J. M. Turney, T. D. Crawford, and C. D. Sherrill, J. Chem. Theory Comput., 2017, 13, 3185-3197, DOI: 10.1021/acs.jctc.7b00174.

${ }^{69}$ S. C. C. van der Lubbe and C. Fonseca-Guerra, The Nature of Hydrogen Bonds: A Delineation of the Role of Different Energy Components on Hydrogen Bond Strengths and Lengths. Chem. Asian. J., 2019, 14, 27602769, DOI: 10.1002/asia.201900717. 
${ }^{70}$ E. Espinosa, I. Alkorta, J. Elguero and E. Molins, From Weak to Strong Interactions: A Comprehensive Analysis of the Topological and Energetic Properties of the Electron Density Distribution Involving X-H $\cdots \mathrm{F}-$ Y Systems. J. Chem. Phys., 2002, 117, 5529, DOI: 10.1063/1.1501133.

${ }^{71}$ D. Cremer and E. Kraka, A Description of the Chemical Bond in Terms of Local Properties of Electron Density and Energy, Croatica Chimica Acta, 1984, 57, 1259-1281

${ }^{72}$ M. Raupach and R. Tonner, A periodic energy decomposition analysis method for the investigation of chemical bonding in extended systems. J. Chem. Phys., 2015, 142, 194105, DOI: 10.1063/1.4919943. 